\title{
Integration of the Scientific Recommender System Mr. DLib into the Reference Manager JabRef
}

\author{
Stefan Feyer ${ }^{1}$, Sophie Siebert ${ }^{2}$, Bela Gipp ${ }^{1}$, Akiko Aizawa ${ }^{3}$, \\ and Joeran Beel ${ }^{3,4(\bowtie)}$ \\ 1 University of Konstanz, Konstanz, Germany \\ \{stefan.feyer, bela.gipp\}@uni-konstanz.de \\ 2 Otto-von-Guericke University Magdeburg, Magdeburg, Germany \\ sophie.siebert@st.ovgu.de \\ 3 National Institute of Informatics (NII), Tokyo, Japan \\ \{aizawa, beel\}@nii.ac.jp \\ 4 Trinity College Dublin, SCSS, KDEG, ADAPT Centre, Dublin, Ireland \\ joeran. beel@adaptcentre.ie
}

\begin{abstract}
This paper presents a description of integration of the Mr. DLib scientific recommender system into the JabRef reference manager. Scientific recommender systems help users identify relevant papers out of vast amounts of existing literature. They are particularly useful when used in combination with reference managers. Over $85 \%$ of JabRef users stated that they would appreciate the integration of a recommender system. However, the implementation of literature recommender systems requires experience and resources that small companies cannot afford. With the desires of users in mind, we integrated the Mr. DLib scientific recommender system into JabRef. Using Mr. DLib's recommendationsas-a-service, JabRef users can find relevant literature and keep themselves informed about the state of the art in their respective fields.
\end{abstract}

Keywords: IR · Scientific recommender system • Reference manager • Recommendations-as-a-service

\section{Introduction}

Scientific recommender systems are used by many academic services, including digital libraries and reference managers. Reference managers are particularly suitable for offering recommendations on related articles because such recommendations enable users to identify relevant information more easily from the large volume of existing reports, articles, and papers. This service not only provides access to local documents; it supports exploration of the relevant documents in external databases such as SowiPort and the ACM digital library.

Although Mendeley, ReadCube, and Docear [4] already have integrated recommender systems, JabRef does not have one. With over 2.5 million downloads 
over the past 13 years $^{1}$, JabRef has become an exceedingly popular reference manager ${ }^{2}$. According to a 2015 survey $^{3}$ yielding more than 428 responses, JabRef has users of 29 different languages, mostly German (42\%) and English (26\%). Respondents stated that they were using JabRef for their professional work $(56 \%)$, but also for their studies and personal work. The largest field of interest among JabRef users was the natural sciences (36\%), but formal sciences (21\%), social sciences (10\%), and humanities $(7 \%)$ also commanded interest from users. Over $85 \%$ of users stated that a recommender system, if one were integrated into JabRef, would be useful. However, JabRef is developed and run by volunteers with limited time. Additionally, the team has little expertise in recommender systems, and lacks even the limited resources (e.g. servers) necessary to implement a recommender system. Therefore, recommendations-as-a-service, a recommendation service for which a third party is responsible for hosting a recommender system, is particularly attractive for JabRef. In the case of JabRef, it would request recommendations from a third party via an API.

For academic use, BibTip [6] and ExLibris $\mathrm{bX}^{4}$ provide recommendations based on co-occurrence [3]. The CORE recommender [5] uses collaborative filtering and content-based filtering. Babel [7] was recently developed by DataLab, which is part of the Information School at the University of Washington.

Finally, Mr. DLib ${ }^{5}$ (Machine Readable Digital Library) [2] is a free, opensource, RESTful web-service that generates recommendations based on a single document and which offers different recommendation approaches, such as stereotype-based and content-based algorithms with additional re-ranking using bibliometric data ${ }^{6}$. Additionally, Mr. DLib gathers data about the recommendations made. These data are utilized to evaluate the different algorithms, which in the long run will optimize scientific recommender systems. The Mendeley and ReadCube reference managers parse users' libraries and calculate recommendations based on this library. Both are companies with sufficient resources to implement this functionality independently. Docear visualizes the literature in a mind-map, providing several options for displaying additional information, such as annotations and relations to other papers in the mind-map. These mind-maps are utilized for the recommendations using content-based filtering.

The team responsible for JabRef chose to integrate Mr. DLib into the JabRef desktop application because it is open-source and because it includes the CORE dataset, with more than 20 million documents. Another reason Mr. DLib was

${ }^{1}$ https://sourceforge.net/projects/jabref/files/jabref/stats/timeline?dates=2003-10-1 2+to+2015-10-01 and https://www.fosshub.com/JabRef.html, accessed on 2016$10-19$.

2 http://www.docear.org/2013/11/11/on-the-popularity-of-reference-managers-andtheir-rise-and-fall/, accessed on 2016-10-28.

${ }^{3}$ http://www.jabref.org/surveys/2015/analysis, accessed on 2016-10-21.

${ }^{4}$ http://www.exlibrisgroup.com/category/bXRecommender, accessed on 2016-10-19.

${ }^{5}$ http://mr-dlib.org/, accessed on 2016-10-19.

${ }^{6}$ Detailed information about the specific algorithms and their evaluation will be published in the near future. 
chosen is that the recommendation approach of BibTip and ExLibris is mostly used to enrich online catalogues, although it is difficult to integrate.

Our overall goal is to support JabRef users in finding relevant literature and to discover relevant content within the vast amount of existing literature.

\section{System Overview and Implementation}

JabRef, a free open-source application with modest hardware requirements, is a desktop program available for all major operating systems. The user's literature being managed is presented as a table, as shown in Fig. 1 (left). Every row in the table represents a BibTex item. JabRef provides basic reference management tools such as sorting, filtering, and searching. Selecting an item opens a preview in the bottom area and displays basic information related to the BibTex item including the abstract, if it is given. Double-clicking on an entry gives access to detailed information related to the paper and the BibTex file itself through the so-called Entry Editor, which also accommodates editing of the file.

Multiple possibilities presented themselves for displaying the recommendations in the existing JabRef GUI. On the one hand, the area for showing literature recommendations should be highly visible, but on the other hand, it should fit into the existing structure of JabRef. One possible solution was to place recommendations in the preview of a BibTex entry. We considered creating a new section in the preview showing a list of the recommended articles. An alternative was placing a button in this preview, which opens a pop-up containing the recommendations. However, pop-ups would break with the existing structure of JabRefs GUI. In addition, a short evaluation of the user behavior revealed that this preview was not frequently used. Instead, users were using the Entry Editor. After considering these alternatives, we chose to add a new tab called Related articles in the Entry Editor, as shown in Fig. 1 (right). To place it as a new tab in this section fits into the existing structure. It does not feel intrusive and it is easily visible.

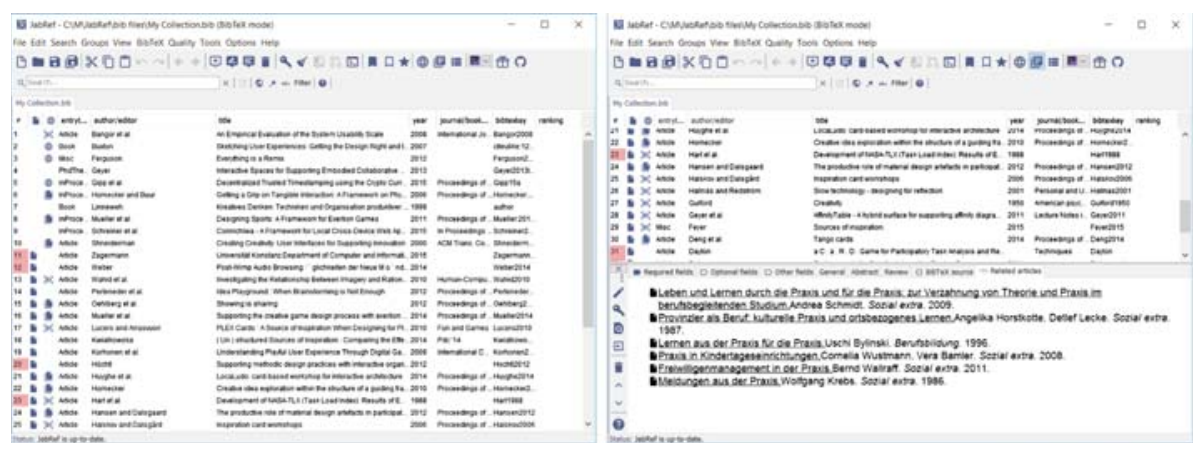

Fig. 1. (left) The JabRef GUI. No entry selected. (right) JabRef GUI with the new Related articles tab at the bottom. Six recommendations are displayed. 
Whenever a user selects an entry, the title of the entry is transferred to Mr. DLib. Using this title, Mr. DLib calculates related articles and responds with an XML file containing the recommendations. This response is parsed by the JabRef application and is then displayed in the Related articles tab, which takes about one second. During this second, a loading animation is displayed in the tab, indicating that a request for the selected entry is in progress.

A click on the link of the recommendation produces a new browser tab, where the PDF can be downloaded. In addition, new recommendations based on the selected article are shown on this site, inviting users to continue browsing.

The application will become available online with the next update of JabRef. The current version is accessible on GitHub.

\section{Outlook}

The current version of Mr. DLib is specified to deliver recommendations based on a single document. Future versions will make it possible to calculate recommendations using a set of documents and to provide recommendations based on this set. Furthermore, Mr. DLib is constantly extending its database with new documents. The gathered data about the displayed recommendations, e.g. if they were clicked inside the Desktop application of JabRef, are expected to contribute to the improvement of academic literature recommender systems in the long run. The integration of Mr. DLib into JabRef has now enabled the Mr. DLib developers to conduct further research on reproducibility in recommendersystems research [1].

Acknowledgements. This publication has derived from research conducted with the financial support of Science Foundation Ireland (SFI) under Grant Number 13/RC/2106. We are also grateful for support received by Siddharth Dinesh and the JabRef Team: Oliver Kopp, Simon Harrer, Joerg Lenhard, Stefan Kolb, Matthias Geiger, Oscar Gustafsson, Tobias Diez, and Christoph Schwentker.

\section{References}

1. Beel, J., Breitinger, C., Langer, S., Lommatzsch, A., Gipp, B.: Towards reproducibility in recommender-systems research. User Model. User-Adap. Inter. (UMUAI) 26(1), 69-101 (2016)

2. Beel, J., Gipp, B., Langer, S., Genzmehr, M., Wilde, E., Nürnberger, A., Pitman, J.: Introducing Mr. DLib, a machine-readable digital library. In: Proceedings of the 11th Annual International ACM/IEEE Joint Conference on Digital Libraries, pp. 463-464. ACM (2011)

3. Beel, J., Langer, S.: A comparison of offline evaluations, online evaluations, and user studies in the context of research-paper recommender systems. In: Kapidakis, S., Mazurek, C., Werla, M. (eds.) TPDL 2015. LNCS, vol. 9316, pp. 153-168. Springer, Heidelberg (2015). doi:10.1007/978-3-319-24592-8_12

4. Beel, J., Langer, S., Genzmehr, M., Nürnberger, A.: Introducing Docear's research paper recommender system. In: Proceedings of the 13th ACM/IEEE-CS Joint Conference on Digital Libraries - JCDL 2013, p. 459 (2013) 
5. Knoth, P.: Linking textual resources to support information discovery. Ph.D. thesis, The Open University (2015)

6. Mönnich, M., Spiering, M.: Erschließung. Einsatz von BibTip als Recommendersystem im Bibliothekskatalog. Bibliotheksdienst 42(1), 54-59 (2008)

7. Wesley-Smith, I., West, J.D.: Babel: a platform for facilitating research in scholarly article discovery. In: Proceedings of the 25th International Conference Companion on the World Wide Web, pp. 389-393 (2016) 